\title{
Dietary-lifestyle patterns associated with adiposity and metabolic abnormalities in young men: cross-sectional study (MeDiSH Project)
}

\author{
Marta Lonnie $^{1}$, Lidia Wadolowska ${ }^{1}$, Joanna Kowalkowska ${ }^{1}$ and \\ Elzbieta Bandurska-Stankiewicz ${ }^{2}$ \\ ${ }^{1}$ Faculty of Food Sciences, University of Warmia and Mazury in Olsztyn, Olsztyn, 10-718, Poland and \\ ${ }^{2}$ Faculty of Medical Sciences, University of Warmia and Mazury in Olsztyn, Olsztyn, 10-718, Poland
}

\begin{abstract}
The aim of this study was to examine the associations of dietary-lifestyle patterns (DLPs) with adiposity and metabolic abnormalities in young Polish men. The cross-sectional study included 367 men 19-40-year-old. Dietary and lifestyle behaviours were determined with food frequency questionnaire (Jezewska-Zychowicz et al. 2018, http://www.knozc.pan.pl). DPLs were derived with Principal Component Analysis. Body size and composition was assessed using measuring tapes and bioelectrical impedance analysis (BIA) method. Adiposity was determined by the assessment of excessive body weight (body mass index, BMI $=25-29.9 \mathrm{~kg} / \mathrm{m}^{2}$ for overweight and $\geq 30 \mathrm{~kg} / \mathrm{m}^{2}$ for obesity), body-fat content (percentage body fat, $\% \mathrm{BF}>25 \%$ ), central obesity status (waist circumference, WC $>$ $102 \mathrm{~cm})$ and skeletal muscle mass $\left(\mathrm{SMM}<31 \mathrm{~kg} / \mathrm{m}^{2}\right)$. Metabolic abnormalities were determined if parameters exceeded: $100 \mathrm{mg} / \mathrm{dL}$ for fasting blood glucose (FBG), 150mg/dL for triglycerides (TG), $200 \mathrm{mg} / \mathrm{dL}$ for total cholesterol (TC) and at least one component of blood pressure (BP) was above the norm ( $\mathrm{SBP} \geq 130 \mathrm{mmHg}$ or/and $\mathrm{DBP} \geq 80 \mathrm{mmHg}$ ). Multivariate logistic regression was used to calculate odds ratio (OR) and verify the association between variables. Four DLPs were derived, explaining 33\% of the variance. Greater adherence (upper vs. bottom tertile) to "Protein food, fried-food and recreational physical activity" (DLP1) and "Healthy diet, activity at work, former smoking" (DLP4) patterns was associated with higher odds of being overweight (odds ratio, OR $=2.12,95 \%$ confidence interval, 95\%CI: 1.15-3.89; 3.05,1.69-5.53) but with high SMM $(2.62,1.53-4.49 ; 3.27,1.91-5.59)$ and lower odds of central obesity $(0.36,0.16-0.83 ; 0.30,0.12-0.74)$ and high body-fat content $(0.22,0.11-0.43 ; 0.37,0.19-0.72)$. In addition, men from the upper tertile of DLP1 had lower odds of increased TC $(0.43,0.24-0.75)$. Greater adherence to "Sandwiches and convenience foods" pattern (DPL2) was associated with higher odds of central obesity (3.36,1.38-8.12), high body-fat content (3.69, 1.88-7.24) and high TC (2.50, 1.47-4.59) and lower odds of high SMM (0.54,0.32-0.90). Greater adherence to "Fast foods and stimulants" pattern (DLP3) was associated with higher odds of general and central obesity $(2.56,1.00-6.56 ; 3.54,1.53-8.19)$, high body-fat content $(4.47,2.05-9.73)$, but not with metabolic abnormalities. No associations between upper tertiles of DLPs and FBG, TG and BP were found. The clustering of dietary and lifestyle behaviours in men revealed that healthy diet attempts combined with active lifestyle, at work or leisure time, reduced risk of adiposity and metabolic abnormalities, despite some unhealthy components, former smoking or fried-food consumption. The study strengthens previous findings that unhealthy dietary behaviours have an adverse effect on adiposity outcomes and metabolic health, potentially through the mechanisms associated with central obesity.
\end{abstract}

\section{Conflict of Interest}

There is no conflict of interest. Project financially supported by Minister of Science and Higher Education in the range of the program entitled "Regional Initiative of Excellence" for the years 2019-2022, Project No. 010/RID/2018/19, amount of funding 12.000.000 PLN. 